\title{
Structural and electronic properties of monolayer group III monochalcogenides
}

\author{
S. Demirci ${ }^{1,2}$ N. Avazl $1,{ }^{3}$ E. Durgun, ${ }^{1}$ and S. Cahangirov ${ }^{1, *}$ \\ ${ }^{1}$ UNAM-Institute of Materials Science and Nanotechnology, Bilkent University, 06800 Ankara, Turkey \\ ${ }^{2}$ Department of Physics, Kırlkkale University, Kırlkkale 71450, Turkey \\ ${ }^{3}$ Department of Physics, Bilkent University, Ankara 06800, Turkey \\ (Received 21 November 2016; revised manuscript received 6 February 2017; published 6 March 2017)
}

\begin{abstract}
We investigate the structural, mechanical, and electronic properties of the two-dimensional hexagonal structure of group III-VI binary monolayers, $M X(M=\mathrm{B}, \mathrm{Al}, \mathrm{Ga}$, In and $X=\mathrm{O}, \mathrm{S}, \mathrm{Se}, \mathrm{Te})$ using first-principles calculations based on the density functional theory. The structural optimization calculations and phonon spectrum analysis indicate that all of the 16 possible binary compounds are thermally stable. In-plane stiffness values cover a range depending on the element types and can be as high as that of graphene, while the calculated bending rigidity is found to be an order of magnitude higher than that of graphene. The obtained electronic band structures show that $M X$ monolayers are indirect band-gap semiconductors. The calculated band gaps span a wide optical spectrum from deep ultraviolet to near infrared. The electronic structure of oxides $(M O)$ is different from the rest because of the high electronegativity of oxygen atoms. The dispersions of the electronic band edges and the nature of bonding between atoms can also be correlated with electronegativities of constituent elements. The unique characteristics of group III-VI binary monolayers can be suitable for high-performance device applications in nanoelectronics and optics.
\end{abstract}

DOI: 10.1103/PhysRevB.95.115409

\section{INTRODUCTION}

Graphene, a two-dimensional (2D) monolayer of carbon atoms arranged in a honeycomb lattice, is intensively studied both theoretically and experimentally due to its impressive mechanical properties, high carrier mobility, wide band absorption, and other exotic behaviors mainly originating from its electronic bands with linear dispersion at the Fermi level (Dirac cones) [1-3]. However, in its pristine semimetallic form, graphene has limited functionality in the semiconductor technology. This motivated research on semiconducting $2 \mathrm{D}$ materials including graphene derivatives such as graphane [4], fluorographane [5], and other systems including metal dichalcogenides [6-9], oxides [10], etc. The rise of graphene also motivated theoretical studies on monolayers of other group IV systems [11,12]. Although these materials lack a naturally occurring parent layered material (similar to graphite), advanced computational analysis has shown that they have thermally stable freestanding single-layer buckled honeycomb lattice. These theoretical studies were followed by experimental works reporting synthesis of silicene [13], germanene [14], and stanene [15] on various substrates. Furthermore, a field-effect transistor based on silicene was realized recently and reported to operate, although for a short time, at room temperature [16].

The experimental verifications of theoretically predicted materials fueled computational studies that explore the periodic table even further to reveal other stable 2D structures with novel properties. These investigations resulted in the discovery of many 2D families such as group IV and group III-V binary compounds in honeycomb structure [17]. Very recently, theoretical studies have reported a stable class of single-layer group IV monochalcogenides $(Y X, Y=\mathrm{Si}$, Ge, $\mathrm{Sn}$ and $X=\mathrm{S}, \mathrm{Se}$, and $\mathrm{Te}$ ) that are semiconductors with

\footnotetext{
*seymur@unam.bilkent.edu.tr
}

wide band gaps [18-20]. Furthermore, a study focused on the structural and electronic properties of these systems reported indirect-direct band gap transitions under small mechanical strain [19]. Most recently, the transition from semiconductor to metal has been achieved when a large compressive strain was applied to monolayers of $\mathrm{Ge} X$ and $\operatorname{Sn} X$ [21]. The possibility of tuning electronic structure is critical for applications in nanoelectronics and optoelectronics. Nonetheless, the detailed chemical and physical mechanisms leading to the observed structural and electronic properties of these 2D monolayers are yet to be understood.

Another interesting and new family of $2 \mathrm{D}$ materials is monolayer group III metal chalcogenides, $M X$, where $M$ (metal) is $\mathrm{B}, \mathrm{Ga}, \mathrm{Al}$, or In and $X$ (chalcogen) is $\mathrm{O}, \mathrm{S}, \mathrm{Se}$, or Te. To date, various $M X$ systems such as InSe [22,23], GaS [24-26], GaSe [27,28], GaTe [29], and $\mathrm{In}_{2} \mathrm{Se}_{3}$ [30,31] have been experimentally realized. Besides the fabrication, great endeavor has been dedicated to reveal the intriguing electronic and optical properties of $M X$ and their potential applications for use in many fields [32]. Among these, monolayer GaS and $\mathrm{GaSe}$ are great candidates for being used in photodetectors due to their strong absorption behavior in the UV-visible wavelength $[27,33]$. GaS sheets also have strong and unique photoresponse behavior in different gaseous environments [24]. In a theoretical study, single layer GaS and GaSe were suggested as potential photocatalysts for water splitting when the band edge position is compared with the redox potential of water [34]. It has been shown both experimentally and theoretically that the band gap and optical properties of $\mathrm{Ga} X$ can be controlled by mechanical deformation [35]. In addition, the electronic properties of $\mathrm{GaS} / \mathrm{GaSe}$ heterostructures could also be tuned by strain and vertically stacking of $\mathrm{GaS}$ and $\mathrm{GaSe}$ single leads to splitting of electrons and holes [36]. Another layered $M X$ system, InSe, also presents a direct-to-indirect band gap transition when the sample thickness decreases [23]. Interestingly, this is an opposite trend when compared to transition-metal dichalcogenides, such as $\operatorname{MoS}_{2}[7,37]$. As a 
result, a few-layer InSe shows different photoluminescence performance compared to $\mathrm{MoS}_{2}$. Despite these efforts, studies focused only on $\mathrm{Ga}$ and In as the group III element and $\mathrm{S}$, Se, and Te as the group VI element; remaining configurations, especially $M O$ systems, have not been studied yet.

In this paper, we investigate the structural, mechanical, and electronic properties of group III-VI monolayers, $M X(M$ : $\mathrm{B}, \mathrm{Al}, \mathrm{Ga}$, In and $X: Y=\mathrm{O}, \mathrm{S}, \mathrm{Se}, \mathrm{Te})$ using first-principles techniques. This enables systematic analysis and comparison of all possible configurations. In the first part, the relevant structural parameters are obtained and cohesive energies are reported. Next, the stability of these systems is investigated using phonon spectrum analysis. In the mechanical properties section, elastic stiffness, Poisson's ratio, and bending rigidities are attained. Finally, the electronic band structures are studied and corresponding band gaps are calculated. We reveal the trends in obtained properties of all systems depending on the constituent atoms and, in particular, their electronegativities $(\chi)$.

\section{METHODS}

We performed first-principles calculations based on the density functional theory (DFT) using a plane-wave basis set with an energy cutoff ranging from $230 \mathrm{eV}$ (for InTe) to $520 \mathrm{eV}$ (for $M \mathrm{O}$ ). We utilize projector augmented-wave potentials [38] and the exchange-correlation potential is approximated by generalized gradient approximation (GGA) with the Perdew, Burke, and Ernzerhof (PBE) functional [39]. The Brillouin zone (BZ) is represented by a $16 \times 16 \times 1 k$-point grid in the Monkhorst-Pack scheme [40]. The equilibrium configuration of atoms is determined by minimizing the total energy of the system using the conjugate gradient method. The energy convergence criteria between the two consecutive electronic and ionic steps are taken to be $10^{-5}$ and $10^{-4} \mathrm{eV}$, respectively. To avoid interaction between periodic images in adjacent cells, a vacuum spacing of at least $12 \AA$ was used. All calculations are performed using the Vienna Ab initio Simulation Package (VASP) [41-44]. The effective charge on atoms is analyzed using the Bader method [45]. We also apply Heyd-ScuseriaErnzerhorf (HSE06) hybrid functionals [46] for selected cases to obtain corrected band gap values. The HSE06 functional is constructed by mixing $25 \%$ of the Fock exchange with $75 \%$ of the PBE exchange and $100 \%$ of the PBE correlation.

We calculate the phonon spectrum using a small displacement method. The force constant matrix is constructed by slight displacement of atoms in a $6 \times 6 \times 1$ supercell. PHON software [47] was used to determine the necessary displacements and to calculate the phonon dispersions using the obtained force constants.

Cohesive energy $\left(E_{C}\right)$ of the system is calculated using the following expression:

$$
E_{C}=\left[\left(N_{M} E_{M}+N_{X} E_{X}\right)-E_{M X}\right] /\left(N_{M}+N_{X}\right),
$$

where $E_{M X}$ is the total energy of the system, while $E_{M}$ and $E_{X}$ are the single atom energies of the constituent elements $M$ and $X$, which stand for the group III and group VI atoms, respectively. $N_{M}\left(N_{X}\right)$ is the number of $M(X)$ atoms in the unit cell.

\section{RESULTS AND DISCUSSION}

\section{A. Atomic structure and energetics}

The model system of $M X$ monolayers (with $M=\mathrm{B}, \mathrm{Al}$, $\mathrm{Ga}$, In and $X=\mathrm{O}, \mathrm{S}, \mathrm{Se}, \mathrm{Te}$ ) is constructed according to the geometry of monolayer GaSe and $\mathrm{GaS}$, which have already been synthesized [26,27]. All 16 possible combinations of $M X$ compounds form honeycomb structures which consist of four-atom basis in a unit cell with four sublayer stacking in the order of $X-M-M-X$ as demonstrated in Fig. 1(a). This geometry belongs to the space group $D_{3 h}$. By cutting the covalent $M-M$ bonds one can get two layers that have the same geometry as the buckled group III-V monolayers [17]. However, the experimental data indicate that $M X$ systems grow in the "bilayer" geometry.

The calculated structural properties are summarized in Table I. The obtained results for $\mathrm{Ga} X$ and $\operatorname{In} X(X=\mathrm{S}, \mathrm{Se}$, Te) are in good agreement with previous theoretical studies [34,36,48-50]. The bond length between $M$ and $X$ atoms, $d_{M X}$, increases when moving down in a group and/or moving across a period in parallel with the increase in the atomic radius of an $M$ and/or an $X$ element. The lattice constant, $a$, follows a similar trend since it is proportional to $d_{M X}$. On the other hand, the bond length between $M$ atoms, $d_{M M}$, increases as one moves down in group and does not depend on the type of $X$ atoms. The bond angle $\theta(\angle M X M$ or $\angle X M X)$ decreases as the period of the $X$ atom is increased and shows small variation with respect to the $M$ element. The thickness of the monolayer (h) is proportional to both $d_{M X}$ and $d_{M M}$ and hence follows the corresponding trend (vide supra).
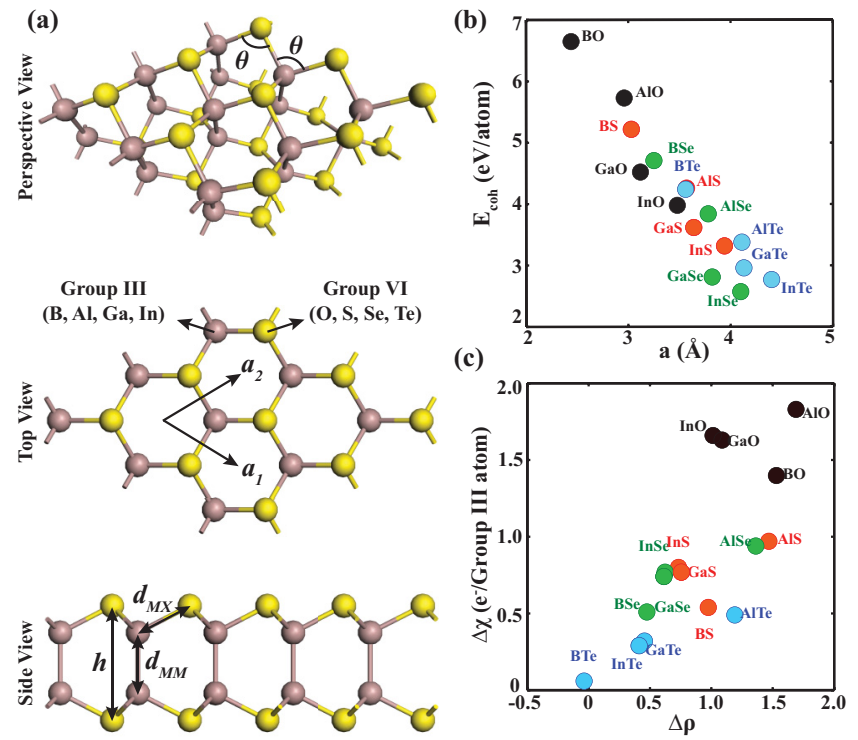

FIG. 1. (a) Perspective, top and side views of the structures of the group III-VI monolayers. The unit cell lattice vectors $\boldsymbol{a}_{\mathbf{1}}$ and $\boldsymbol{a}_{\mathbf{2}}$ are equal and have the same length $a$. The bond lengths ( $d_{M M}$ and $\left.d_{M X}\right)$ and height $(h)$ are also shown. (b) The variation of cohesive energies $\left(E_{C}\right)$ with the lattice constants, $a$, of $M X$ systems. (c) The variation of electronegativity differences $(\Delta \chi)$ between $M$ and $X$ atoms with the charge transfers $(\Delta \rho)$ between them. 
TABLE I. Structural, mechanical, and electronic properties of $M X$ monolayers ( $M X, M=\mathrm{B}, \mathrm{Al}, \mathrm{Ga}$, and $\mathrm{In}, X=\mathrm{O}, \mathrm{S}, \mathrm{Se}$, and Te): lattice constant $(a)$, bond length $\left(d_{M X}\right.$ and $\left.d_{M M}\right)$, thickness $(h)$, bond angle $(\theta)$, cohesive energy $\left(E_{C}\right)$, band gap $\left(E_{G}\right)$, Poisson's ratio $(v)$, in-plane stiffness $(C)$, bending rigidity $(D)$, electronegativity difference $(\Delta \chi)$, and charge transfer $(\Delta \rho)$. Structural parameters are described in Fig. 1 , and electronic band structures are detailed in Figs. 3 and 4.

\begin{tabular}{|c|c|c|c|c|c|c|c|c|c|c|c|c|}
\hline$M X$ & $\begin{array}{c}a \\
(\AA)\end{array}$ & $\begin{array}{r}d_{M X} \\
(\AA)\end{array}$ & $\begin{array}{r}d_{M M} \\
(\AA)\end{array}$ & $\begin{array}{c}h \\
(\AA)\end{array}$ & $\begin{array}{c}\theta \\
(\operatorname{deg})\end{array}$ & $\begin{array}{c}E_{C} \\
\text { (eV/atom) }\end{array}$ & $\begin{array}{l}E_{G} \\
(\mathrm{eV})\end{array}$ & $v$ & $\begin{array}{c}C \\
\left(\mathrm{~J} / \mathrm{m}^{2}\right)\end{array}$ & $\begin{array}{c}D \\
(\mathrm{eV})\end{array}$ & $\Delta \chi$ & $\begin{array}{c}\Delta \rho \\
\left(\mathrm{e}^{-}\right)\end{array}$ \\
\hline BO & 2.44 & 1.52 & 1.77 & 2.92 & 106.7 & 6.65 & $4.71 / M \Gamma-M$ & 0.20 & 350 & 30 & 1.40 & 1.52 \\
\hline $\mathrm{AlO}$ & 2.96 & 1.83 & 2.62 & 3.94 & 107.8 & 5.73 & $1.30 / K-M$ & 0.37 & 149 & 27 & 1.83 & 1.69 \\
\hline $\mathrm{GaO}$ & 3.12 & 1.94 & 2.51 & 3.99 & 106.5 & 4.52 & $1.54 / \Gamma K-\Gamma$ & 0.40 & 130 & 26 & 1.63 & 1.08 \\
\hline $\mathrm{InO}$ & 3.48 & 2.16 & 2.86 & 4.47 & 107.0 & 3.98 & $0.45 / K-\Gamma$ & 0.44 & 75 & 24 & 1.66 & 1.01 \\
\hline BS & 3.03 & 1.94 & 1.72 & 3.42 & 102.4 & 5.22 & $2.88 / M \Gamma-M$ & 0.12 & 212 & 22 & 0.54 & 0.97 \\
\hline AlS & 3.57 & 2.32 & 2.59 & 4.73 & 100.4 & 4.26 & $2.10 / \Gamma K-M$ & 0.25 & 80 & 16 & 0.97 & 1.46 \\
\hline $\mathrm{GaS}$ & 3.64 & 2.36 & 2.47 & 4.65 & 100.4 & 3.62 & $2.35 / \Gamma K-\Gamma$ & 0.22 & 73 & 16 & 0.77 & 0.75 \\
\hline InS & 3.94 & 2.56 & 2.82 & 5.19 & 100.2 & 3.32 & $1.64 / \Gamma K-\Gamma$ & 0.31 & 50 & 14 & 0.80 & 0.73 \\
\hline BSe & 3.25 & 2.10 & 1.71 & 3.60 & 101.3 & 4.71 & $2.61 / \Gamma-M$ & 0.15 & 172 & 16 & 0.51 & 0.47 \\
\hline $\mathrm{AlSe}$ & 3.78 & 2.47 & 2.57 & 4.90 & 99.75 & 3.84 & $1.99 / \Gamma K-M$ & 0.24 & 66 & 20 & 0.94 & 1.36 \\
\hline GaSe & 3.82 & 2.50 & 2.46 & 4.81 & 99.77 & 2.81 & $1.77 / \Gamma K-Г$ & 0.24 & 67 & 13 & 0.74 & 0.61 \\
\hline InSe & 4.10 & 2.69 & 2.81 & 5.37 & 99.26 & 2.57 & $1.37 / \Gamma K-\Gamma$ & 0.29 & 42 & 11 & 0.77 & 0.62 \\
\hline BTe & 3.56 & 2.31 & 1.71 & 3.82 & 100.8 & 4.24 & $1.52 / \Gamma-M$ & 0.15 & 136 & 15 & 0.06 & -0.03 \\
\hline $\mathrm{AlTe}$ & 4.11 & 2.70 & 2.58 & 5.14 & 99.30 & 3.38 & $1.84 / M \Gamma-M$ & 0.23 & 54 & 14 & 0.49 & 1.19 \\
\hline GaTe & 4.13 & 2.70 & 2.46 & 5.02 & 99.56 & 2.96 & $1.43 / M \Gamma-M$ & 0.20 & 55 & 14 & 0.29 & 0.41 \\
\hline InTe & 4.40 & 2.89 & 2.81 & 5.86 & 98.95 & 2.77 & $1.29 / \Gamma K-Г$ & 0.23 & 39 & 9 & 0.32 & 0.45 \\
\hline
\end{tabular}

Our results indicate that $E_{C}$ which is calculated using Eq. (1) decreases when going down in a group (and/or moving across a period) following the same trend with $d_{M X}$. For a given pair of $M$ and $X$ elements, the shorter $d_{M X}$ is, the stronger the binding of the system. The variation of $E_{C}$ is also portrayed as a function of $a$ in Fig. 1(b). Energetically from the most stable structure $\mathrm{BO}\left(E_{C}=6.65 \mathrm{eV}\right)$ to the least stable InTe $\left(E_{C}=2.77 \mathrm{eV}\right)$, the distance of valence electrons from the nucleus for $\mathrm{B}\left[2 s^{2} 2 p\right], \mathrm{O}\left[2 s^{2} 2 p^{4}\right]$ and $\operatorname{In}\left[5 s^{2} 5 p\right], \mathrm{Te}\left[5 s^{2} 5 p^{4}\right]$ increases and so does $a$ of the structure.

It is also important to compare the cohesive energies of oxides with their well known bulk counterparts. As an example, we calculated the cohesive energy of bulk $\mathrm{Al}_{2} \mathrm{O}_{3}$ that has $12 \mathrm{Al}$ and $18 \mathrm{O}$ atoms in the unit cell and found it to be $6.47 \mathrm{eV} /$ atom. This is higher than the $5.74 \mathrm{eV} /$ atom cohesive energy of $\mathrm{AlO}$ in $X-M-M-X$ geometry investigated in this paper. However, it is more reasonable to consider the cohesive energies of $\mathrm{Al}_{2} \mathrm{O}_{3}$ surfaces. In this respect, we cut out two surfaces from bulk $\mathrm{Al}_{2} \mathrm{O}_{3}$; the first one has six $\mathrm{Al}$ and six $\mathrm{O}$ atoms in the unit cell and is terminated with $\mathrm{Al}$ atoms on both sides, while the second one has four $\mathrm{Al}$ and nine $\mathrm{O}$ atoms in the unit cell and is terminated with $\mathrm{O}$ atoms on both sides. Upon geometry optimization, the cohesive energies of $\mathrm{Al}$ and O terminated surfaces were found to be 5.49 and $4.93 \mathrm{eV} /$ atom, respectively. These energies are smaller compared to that of AlO in $X-M-M-X$ geometry which supports the stability of oxides in this structure. Note that, the surfaces cut out of bulk $\mathrm{Al}_{2} \mathrm{O}_{3}$ can have reconstructions lowering their energy, but analyzing those reconstructions would be out of the scope of the present study.

$X$ atoms are more electronegative than $M$ atoms. Hence, $X$ attract electrons around $M$ towards the bonding region that lies between the two atoms. As the $\Delta \chi$ between $M$ and $X$ atoms increase, more electrons are accumulated at the bonds. These bonds, in turn, start to repel each other and increase the angle between them. This is especially evident in the oxides having $\theta$ around $107^{\circ}$, while in the rest it is around $100^{\circ}$. In Fig. 1(c) the charge transfer per atom $\Delta \rho$ from $M$ to $X$ elements versus the $\Delta \chi$ is shown (the corresponding data can be found in Table I). It clearly demonstrates that oxides exhibit a distinct character from those of sulphides, selenides, and tellurides. Recently, a similar trend has also been observed in both group IV-VI [19] and group II-VI monolayers [51].

\section{B. Phonons and stability}

The thermal stability of the proposed 2D structures can be tested by calculating their vibrational spectra. Dynamically stable structures are heralded when calculated dispersions of phonon modes have positive square of frequency throughout the BZ. If there is an imaginary frequency, then there is no restoring force against the displacement of atoms along that particular eigenmode. In Fig. 2, we present the dispersion of phonon modes of $M X$ compounds together with eigenmode analysis. There are no imaginary frequencies in the phonon dispersion curves indicating that all of the considered structures are stable. The phonon dispersion of each monolayer has three acoustic and nine optical branches. The three acoustic branches are the in-plane longitudinal acoustic (LA), the transverse acoustic (TA), and the out-of-plane acoustic (ZA) modes. LA and TA modes have linear dispersion, while ZA modes have quadratic dispersion. The curvature of ZA modes can be used to calculate the bending stiffness, as discussed in the next section.

The $D_{3 h}$ symmetry of the $M X$ structures results in nondegenerate and doubly degenerate modes at the $\Gamma$ point. As shown in the rightmost panel of Fig. 2, there are six distinct eigenmodes that can be used to describe the optical spectrum 

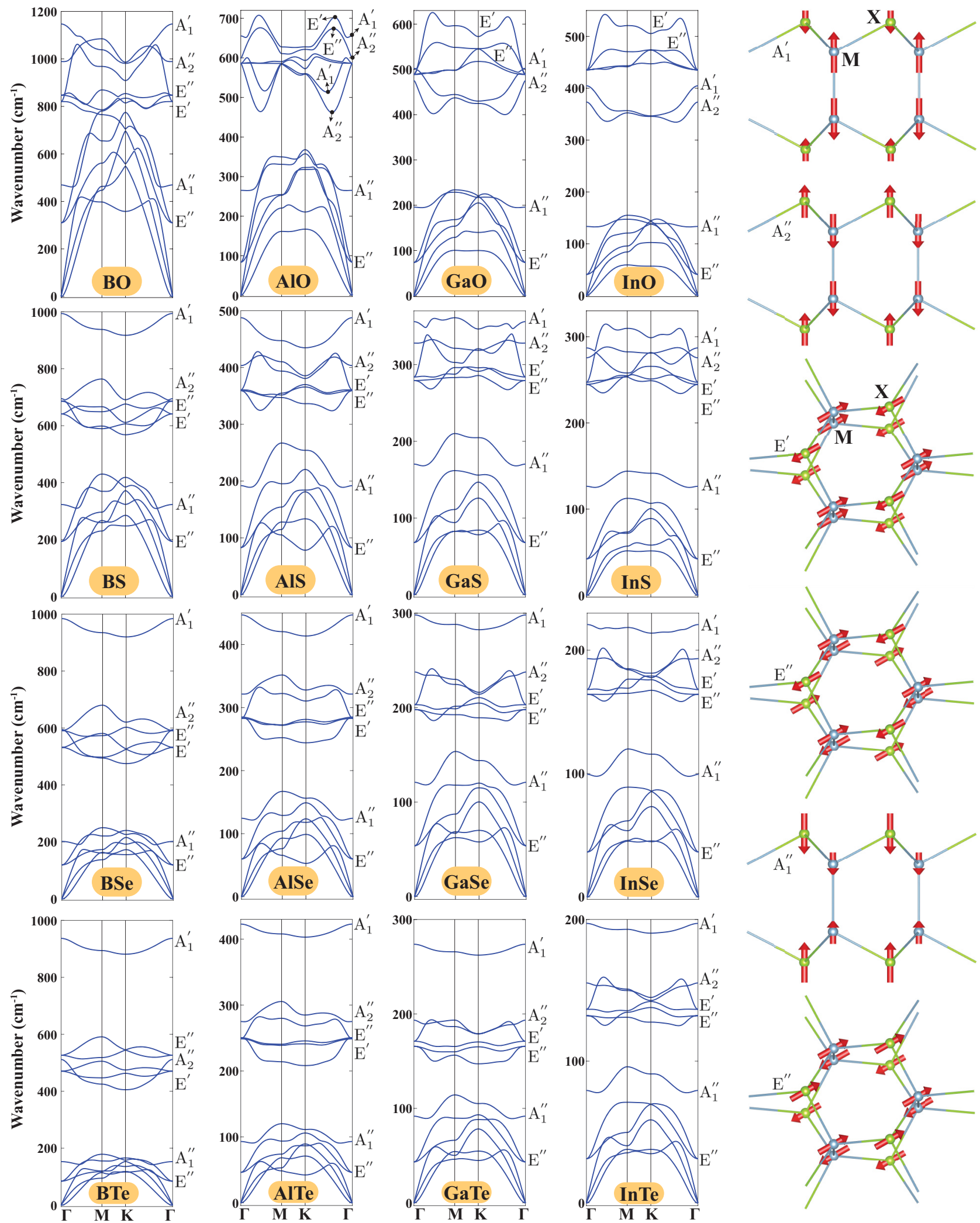

FIG. 2. Phonon dispersions of $M X$ monolayers. The symmetry group representation of eigenvectors at the $\Gamma$ point is shown in the right-hand side of each plot. The representation of specific modes along the $\Gamma-K$ direction is also shown by arrows. Six distinct modes calculated for the InTe structure are shown by ball and stick models. These modes qualitatively define the optical spectrum of all $M X$ structures, although the ratios of the vector magnitudes may vary depending on the $M$ and $X$ atoms. Here, the red arrows correspond to eigenmodes at the $\Gamma$ point. Corresponding symmetry group representations are designated next to each mode. 
of $M X$ structures. The optical mode with lowest energy is the same for all $M X$ structures and it belongs to the $E^{\prime \prime}$ representation. Here the upper $M X$ pair and lower $M X$ pair move opposite to each other in the in-plane direction. In each $M X$ structure there are frequency ranges at which this optical mode coexists together with acoustical modes that leads to the possibility of robust acoustic-optical scattering, and thus a low thermal conductivity. The low-energy $E^{\prime \prime}$ mode is followed by the $A^{\prime \prime}{ }_{1}$ mode in all $M X$ structures. In the $A^{\prime \prime}{ }_{1}$ mode, the upper and lower $M X$ pairs move in opposite out-of-plane directions, in contrast to the opposite in-plane movement of the $E^{\prime \prime}$ mode. The $A^{\prime \prime}{ }_{1}$ mode separates from the $E^{\prime \prime}$ modes and the acoustical modes as the $M$ and $X$ elements become heavier, while it is mixed with these modes when $M$ is $\mathrm{B}$ or $X$ is $\mathrm{O}$. The $A^{\prime \prime}{ }_{1}$ mode touches the higher end of the optical modes only in BO, while for other structures they are separated.

The higher portion of the optical phonon modes of $M X$ structures is composed of six bands. Two of them are nondegenerate and the rest are doubly degenerate at the $\Gamma$ point. In the doubly degenerate $E^{\prime \prime}$ mode (not to be confused with the low-energy $E^{\prime \prime}$ mode discussed above), each of the four atomic planes move in the opposite in-plane directions, while in the doubly degenerate $E^{\prime}$ mode, $M$ atoms and $X$ atoms move in the opposite in-plane directions. Similarly, in the nondegenerate $A^{\prime}{ }_{1}$ mode, each of the four atomic planes moves in the opposite out-of-plane directions, while in the nondegenerate $A^{\prime \prime}{ }_{2}$ mode, $M$ atoms and $X$ atoms move in the opposite out-of-plane directions. In the InO structure, for example, the $A^{\prime}{ }_{1}$ and $A^{\prime \prime}{ }_{2}$ modes are below the $E^{\prime}$ and $E^{\prime \prime}$ modes throughout the $\mathrm{BZ}$, while in the $\mathrm{GaO}$ structure they start to touch. In the AlO structure, this trend is further developed and the $A$ and $E$ modes hybridize. This is clearly seen in the highest optical mode of $\mathrm{AlO}$ that has $A^{\prime}{ }_{1}$ character at the $\Gamma$ point but investigating this eigenmode along the $\Gamma-K$ direction we have found that it develops $E^{\prime}$ character at the peak point (see Fig. 2). These distinctive peaks of the $E^{\prime}$ mode along the $\Gamma-K$ and $\Gamma-M$ directions are also present in $\mathrm{GaO}$ and $\mathrm{InO}$. In the BO structure, the trend is even further developed and the $A$ and $E$ modes switch places.

The $E^{\prime}$ and $E^{\prime \prime}$ modes of oxides have very close energies at the $\Gamma$ point, while they are well separated in the InTe structure. As we change the $M$ atom from In to B (while keeping the Te atom), the gap between the $E^{\prime}$ and $E^{\prime \prime}$ modes closes and they switch their places at the $\Gamma$ point in the BTe structure. Also the dispersions of $E$ modes are significantly different in BTe compared to other tellurides. In this respect, the distinctive peaks of the $E^{\prime}$ mode along the $\Gamma-K$ and $\Gamma-M$ directions fade and disappear. This trend also holds for structures having $\mathrm{S}$ and Se atoms.

The magnitude of the eigenvectors on $M$ atoms are significantly bigger than those on $X$ atoms in the $A^{\prime}{ }_{1}$ mode of InTe, as shown in Fig. 2. These ratios are linearly proportional to the mass ratios of $X$ and $M$ atoms and hence get even bigger in structures having light $\mathrm{B}$ atoms. In fact, we calculated the ratio of the eigenvector magnitudes of the $A^{\prime}{ }_{1}$ mode on $M$ and $X$ atoms to be $2.1,5.2,10.2$, and 16.2 for BO, BS, BSe, and $\mathrm{BTe}$, respectively. The fact that the $A^{\prime}{ }_{1}$ mode is dominated by the movement of $\mathrm{B}$ atoms is mirrored in the minor change in the energy of this mode as we change the $X$ atom from $\mathrm{S}$ to Te.

\section{Mechanical properties}

To investigate the mechanical properties of $M X$ monolayers, we apply compressive and tensile strain up to $1 \%$ in the armchair and zigzag directions denoted by $x$ and $y$, respectively. The atomic coordinates relaxed for each strained case and corresponding energy data are obtained. Assuming that the system is in the harmonic regime, we fit this data to the following quadratic polynomial [17]:

$$
E_{T}\left(\epsilon_{x}, \epsilon_{y}\right)=b_{1} \epsilon_{x}^{2}+b_{2} \epsilon_{y}^{2}+b_{3} \epsilon_{x} \epsilon_{y}+E_{0},
$$

where $E_{0}$ is equilibrium energy per unit cell, $\epsilon_{x}\left(\epsilon_{y}\right)$ is strain in the $x(y)$ direction, $E_{T}\left(\epsilon_{x}, \epsilon_{y}\right)$ is the total energy per unit cell at a strain $\epsilon_{x}$ and $\epsilon_{y}$, and $b_{1}, b_{2}$, and $b_{3}$ are parameters having units of energy. Our calculations show that $b_{1}=b_{2}$, meaning that the elastic response of the material is the same in the armchair and zigzag directions. This behavior is a result of trigonal symmetry and holds true in the harmonic regime [52]. The mechanical properties of $2 \mathrm{D}$ materials are defined by the in-plane stiffness $(C)$ and Poisson's ratio $(v)$, which can be calculated using the following formulas:

$$
\begin{gathered}
C=\left[2 b_{1}-\left(b_{3}\right)^{2} / 2 b_{1}\right] / A_{0}, \\
v=b_{3} / 2 b_{1},
\end{gathered}
$$

where $A_{0}$ is the area of the unit cell at zero strain.

To check the reliability of our calculations, we first determined the $C$ for graphene and found it to be $341 \mathrm{~J} / \mathrm{m}^{2}$, which is in agreement with the corresponding experimental value of $340 \pm 50 \mathrm{~J} / \mathrm{m}^{2}$ [53]. Next we calculated $C$ of all $M X$ binary compounds which are outlined in Table I. Our results indicate that $C$ decreases when moving down in a group and/or moving across a period. Accordingly, the highest $C\left(350 \mathrm{~J} / \mathrm{m}^{2}\right)$ is obtained for BO and this value is comparable with (even slightly larger than) that of graphene. On the other hand, the lowest $C$ is obtained for $\operatorname{InTe}\left(39 \mathrm{~J} / \mathrm{m}^{2}\right)$, which is much smaller than the obtained values for all $M \mathrm{O}$. However, it is comparable with the reported values for phosphorene [54] $\left(C_{x}=26 \mathrm{~J} / \mathrm{m}^{2}\right.$ and $C_{y}=88 \mathrm{~J} / \mathrm{m}^{2}$ ).

To define the mechanical response, we further computed Poisson's ratio $v$, which is the ratio of the transverse strain to the axial strain. In particular, for a given group III element, $v$ decreases almost monotonically when we go from $\mathrm{O}$ to $\mathrm{Te}$ in group VI. As can be noticed, there is a correlation between $C$ and $a$ : the smaller the lattice parameter the larger the in-plane stiffness. In other words, the bonding between the constituent elements becomes weaker leading to smaller in-plane stiffness.

The ZA phonon modes of 2D materials have quadratic dispersion around the $\Gamma$ point [55-57]. As seen in Fig. 2, the $M X$ systems investigated here also possess these modes with quadratic dispersions. In fact, one can obtain an important mechanical property called the bending rigidity, $D$, by calculating the curvature of this quadratic dispersion using the formula derived by Lifshitz [58],

$$
\omega=\sqrt{\frac{D}{\rho_{2 D}}} q^{2},
$$

where $\rho_{2 \mathrm{D}}$ is the $2 \mathrm{D}$ mass density. We first calculated the curvature of the ZA mode of graphene using similar parameters 
and found the $D$ of graphene to be $1.5 \mathrm{eV}$, which is in agreement with the literature [55,56]. Then we considered the ZA modes of the $M X$ structures. Our calculations show that the BO structure, which has the highest in-plane stiffness value, also has the highest bending rigidity, which is around 30 $\mathrm{eV}$. This value is an order of magnitude larger than the value that we calculated for graphene. This means that BO is much more resistant against an out-of-plane distortion compared to graphene. As seen in Table I, other $M X$ structures also have much higher bending rigidity compared to graphene. This tells us that the high bending rigidity is a result of the "bilayer" $X-M-M-X$ geometry that possesses covalent $M-M$ bonds perpendicular to the surface.

\section{Electronic structure}

In this section, we analyze the electronic structure of $M X$ monolayers. We start our analysis by focusing separately on MOs. The electronic band structures of oxides calculated by both DFT-PBE and hybrid functionals (HSE06) [46] are presented in the top panel of Fig. 3. As noticed, all $M O$ monolayers are indirect band gap semiconductors. The band diagrams are not ordered according to the period of $M$ elements
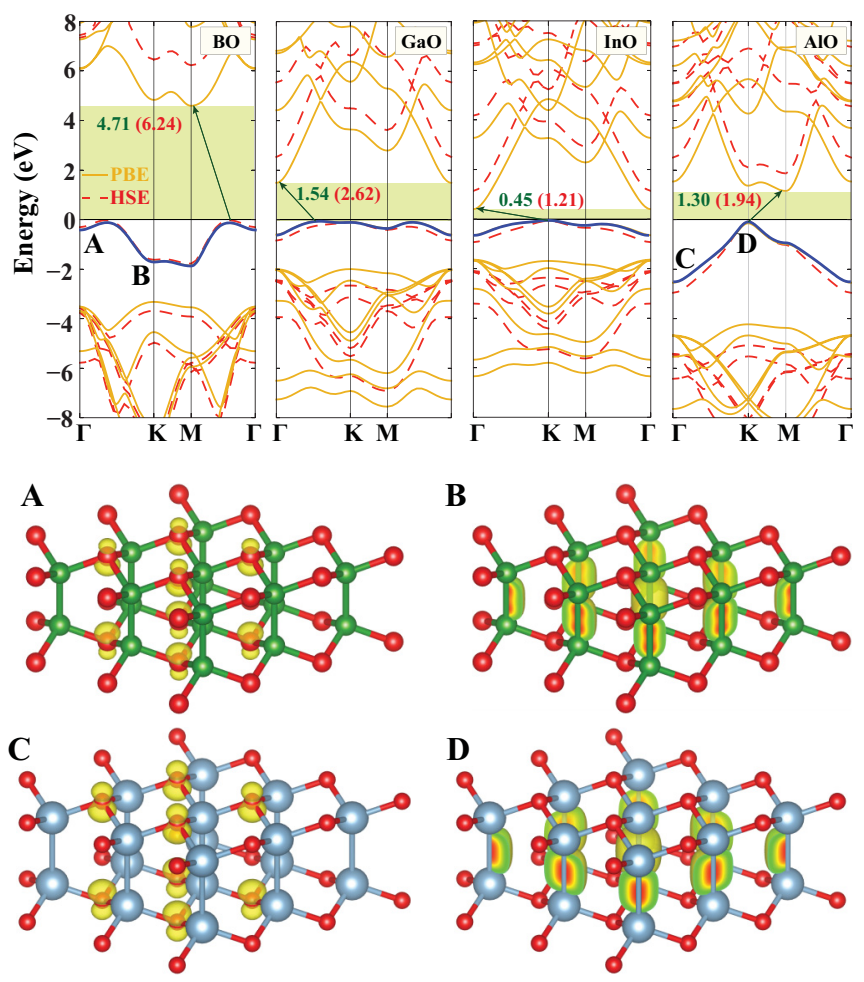

FIG. 3. The electronic band structures of $M O$ (top panel) ordered in the decreasing $\chi(M)$ from left to right. The valence band edges delineated by blue lines are composed of single bands that are isolated from the rest of the filled states. The bands calculated using the PBE and hybrid HSE functionals are shown by solid yellow and dashed red lines, respectively. The energy gap is shaded by the light-green rectangles and the values obtained from PBE and HSE are shown with the dark-green and the red numbers, respectively. The partial charge density isosurfaces of states $A$ and $B$ corresponding to the valence band edge of $\mathrm{BO}$ at the $K$ point and the valence band edge of $\mathrm{AlO}$ at the $\Gamma$ point, respectively, are presented (bottom panel). but instead in the decreasing order of $\chi(M)$, which is 2.04, $1.81,1.78$, and 1.61 for $\mathrm{B}, \mathrm{Ga}$, In, and $\mathrm{Al}$, respectively. This ordering reveals a trend in position of the valence band edge. The electronic states at the $K$ point of valence band edge of BO have significantly lower energy compared to those at the $\Gamma$ point. In the case of $\mathrm{GaO}$ and $\mathrm{InO}$, the electronic states at the $\Gamma$ point are slightly lower than those in the $K$ point. Finally, in AlO this trend is even more enhanced and the states at the $\Gamma$ point have significantly lower energy compared to those at the $K$ point. This trend can be understood by looking at the partial charges presented in the bottom panel of Fig. 3. The electronic states of $\mathrm{BO}$ and $\mathrm{AlO}$ at the $K$ point are accumulated between the $M$ atoms showing a $\sigma$ character. On the other hand, the electronic states at the $\Gamma$ point are accumulated around the $\mathrm{O}$ atoms showing a $\pi$ character. As a result, the states at the $K$ point have lower energy compared to the states at the $\Gamma$ point when the group III element has higher $\chi$.

It is important to note that the highest electronic energy states at the valence band edge of BO shift away from the BZ center ( $\Gamma$ point) [59]. Earlier, these unusual double-peak band maxima have been theoretically predicted for ultrathin $\mathrm{GaS}$ nanosheets which have a thickness less than 5 monolayers [26]. Recently, the distorted band structure of several materials have been examined to understand the parabolic dispersion of the valence band edge at $\Gamma$ deformed into a Mexican-hat dispersion [60,61]. A Mexican-hat dispersion results in ring-shaped valence band edges, which can lead to novel phenomena including a $1 / \sqrt{E}$ singularity in the $2 \mathrm{D}$ density of states $[35,62]$

To reveal any possible computational artifacts, the electronic band structures of $M O$ systems are also calculated using hybrid functionals and compared with DFT-PBE as shown in Fig. 3. Expectedly, the underestimated band gaps increase with this correction and they vary between 1.21 and $6.24 \mathrm{eV}$. On the other hand, overall electronic structure profiles are not affected and hybrid functional correction just shifted the conduction bands to higher energy keeping the dispersions intact.

The electronic band structures of the $M X$ systems beyond oxides are presented in Fig. 4. As the PBE and HSE calculations reveal the same band structure profile, only PBE results are reported. Again, the structures are ordered from left to right according to the decrease in the $\chi(M)$. Including the results of $M \mathrm{O}$, it can be concluded that all $M X$ monolayers are indirect band gap semiconductors. Note that the bulk counterparts of $\mathrm{GaS}, \mathrm{GaSe}$, and InSe are also indirect gap semiconductors with $2.59,2.07$, and $1.35 \mathrm{eV}$ band gaps, respectively [63,64]. The lower and upper limits of the band gap are determined by oxides (vide supra). We also highlight with blue lines the states that behave in the same way as the isolated valence band edges of oxides. Similar to the oxide case, when the blue lines are examined, the states at the $\Gamma$ point lower in energy compared to the states at the $K$ point but the former never dips below the latter like in the $\mathrm{AlO}$ case, because the $\chi$ of $\mathrm{O}$ is much higher than other chalcogenides. In this case, as we go down the period, the $\chi(X)$ decrease from 3.44 for $\mathrm{O}$ down to 2.10 for Te, respectively. As the $\Delta \chi$ (between $M$ and $X$ ) decreases, the blue lines dip down. In fact, BTe is the only structure for which the $\chi(X)$ is higher than that of the $M$ element [see also Fig. 1(c)]; and only in this particular structure (and also in BS but in a much more subtle way) the valence band maximum 


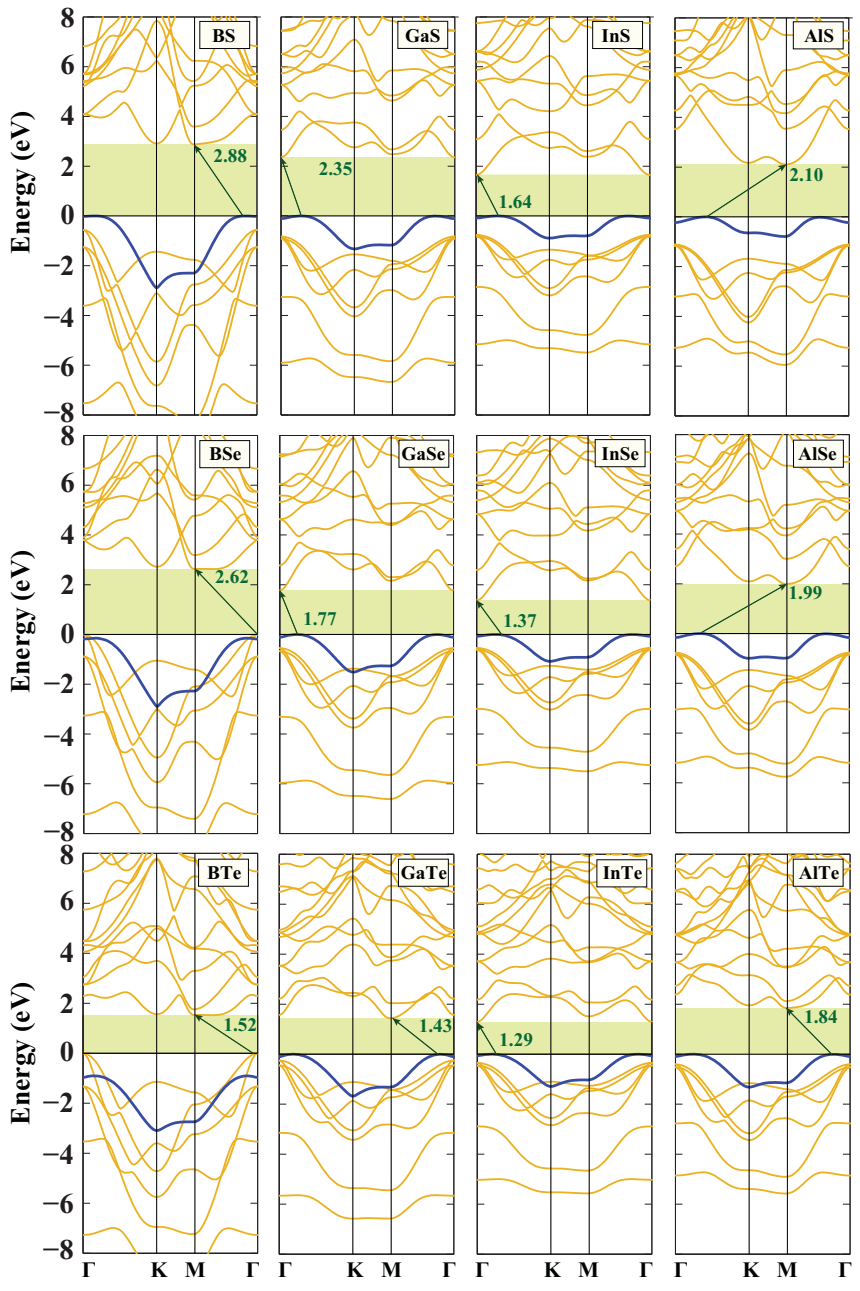

FIG. 4. The electronic band structures of $M X$ monolayers beyond oxides. The structures are ordered in the decreasing $\chi(M)$ from left to right. The bands having the same character as the isolated band edge of oxides are delineated by blue lines, while the rest of the bands are shown by solid yellow lines. The energy gap is shaded by the light-green rectangles and the values (in $\mathrm{eV}$ ) obtained with PBE are given.

is not determined by the blue line. The charge density of the valence band maximum of BTe is accumulated in between the $\mathrm{B}$ and $\mathrm{Te}$ atoms establishing the bonding between them.
Our calculations reveal the Mexican-hat dispersion also in BS, AlS, AlSe, and AlTe while confirming the same observation for GaS, GaSe, GaTe, InS, InSe, and InTe (see Fig. 4). However, the Mexican-hat dispersion is much more pronounced in $\mathrm{BO}$ compared to others.

In general, the energy gaps do not follow a clear trend but they vary between $1.21 \mathrm{eV}(\mathrm{InO})$ and $6.24 \mathrm{eV}(\mathrm{BO})$ covering a range from deep ultraviolet to near infrared parts of the optical spectrum.

\section{CONCLUSION}

In summary, we have investigated the structural, mechanical, and electronic properties of group III monochalcogenides taking into account all possible configurations. The phonon spectrum analysis indicates that all 16 possible structures are thermally stable. We calculated the in-plane stiffness, Poisson's ratio, and the bending rigidity of these structures to characterize the mechanical properties. Our results indicate that in-plane stiffness can be as high as that of graphene and decreases when moving down in a group and/or moving across a period. The bending rigidity of $M O$ systems is very high and can be an order of magnitude larger than that of graphene. The electronic structure calculations show that all $M X$ monolayers are indirect band gap semiconductors, but the upper and lower limits are determined by $M O$. The band gaps span a wide range from deep ultraviolet to near infrared parts of the optical spectrum. We show that BO, BS, AlS, AlSe, and AlTe also possess Mexican-hat dispersion at the valence band edge. These intriguing properties and energy gaps ranging from 1.21 to $6.24 \mathrm{eV}$ makes the predicted structures potential candidates for nanoelectronic applications.

\section{ACKNOWLEDGMENTS}

This work was supported by the Scientific and Technological Research Council of Turkey (TUBITAK) under Project No 115F388. The computational resources are provided by TUBITAK ULAKBIM, High Performance and Grid Computing Center (TR-Grid e-Infrastructure) and the National Center for High Performance Computing of Turkey (UHeM) under Grant No. 5004132016. S.C. acknowledges support from Bilim Akademisi-The Science Academy, Turkey under the BAGEP program.
[1] A. K. Geim and K. S. Novoselov, Nat. Mater. 6, 183 (2007).

[2] F. Schwierz, Nat. Nanotechnol. 5, 487 (2010).

[3] K. S. Novoselov, A. K. Geim, S. V. Morozov, D. Jiang, M. I. Katsnelson, I. V. Grigorieva, S. V. Dubonos, and A. A. Firsov, Nature (London) 438, 197 (2005).

[4] J. O. Sofo, A. S. Chaudhari, and G. D. Barber, Phys. Rev. B 75, 153401 (2007).

[5] J. T. Robinson, J. S. Burgess, C. E. Junkermeier, S. C. Badescu, T. L. Reinecke, F. K. Perkins, M. K. Zalalutdniov, J. W. Baldwin, J. C. Culbertson, P. E. Sheehan, and E. S. Snow, Nano Lett. 10, 3001 (2010).
[6] B. Radisavljevic, A. Radenovic, J. Brivio, V. Giacometti, and A. Kis, Nat. Nanotechnol. 6, 147 (2011).

[7] K. F. Mak, C. Lee, J. Hone, J. Shan, and T. F. Heinz, Phys. Lett. 105, 136805 (2010).

[8] D. Braga, I. G. Lezama, H. Berger, and A. F. Morpurgo, Nano Lett. 12, 5218 (2012).

[9] S. Tongay, W. Fan, J. Kang, J. Park, U. Koldemir, J. Suh, D. S. Narang, K. Liu, J. Ji, J. Li, R. Sinclair, and J. Wu, Nano Lett. 14, 3185 (2014).

[10] D. Loffler, J. J. Uhlrich, M. Baron, B. Yang, X. Yu, L. Lichtenstein, L. Heinke, C. Büchner, M. Heyde, S. 
Shaikhutdinov, H. J. Freund, R. Wlodarczyk, M. Sierka, and J. Sauer, Phys. Lett. 105, 146104 (2010).

[11] S. Cahangirov, M. Topsakal, E. Aktürk, H. Şahin, and S. Ciraci, Phys. Rev. Lett. 102, 236804 (2009).

[12] J. C. Garcia, D. B. de Lima, L. V. C. Assali, and J. F. Justo, J. Phys. Chem. C 115, 13242 (2011).

[13] P. Vogt, P. De Padova, C. Quaresima, J. Avila, E. Frantzeskakis, M. C. Asensio, A. Resta, B. Ealet, and G. Le Lay, Phys. Rev. Lett. 108, 155501 (2012).

[14] C. C. Liu, W. Feng, and Y. Yao, Phys. Rev. Lett. 107, 076802 (2011).

[15] F. Zhu, W. Chen, Y. Xu, C. Gao, D. Guan, C. Liu, D. Qian, S.-C. Zhang, and J. Jia, Nat. Mater. 14, 1020 (2015).

[16] L. Tao, E. Cinquanta, D. Chiappe, C. Grazianetti, M. Fanciulli, M. Dubey, A. Molle, and D. Akinwande, Nat. Nanotechnol. 10, 227 (2015).

[17] H. Şahin, S. Cahangirov, M. Topsakal, E. Bekaroglu, E. Aktürk, R. T. Senger, and S. Ciraci, Phys. Rev. B 80, 155453 (2009).

[18] G. Shi and E. Kioupakis, Nano Lett. 15, 6926 (2015).

[19] C. Kamal, A. Chakrabarti, and M. Ezawa, Phys. Rev. B 93, 125428 (2016).

[20] I. Appelbaum and P. Li, Phys. Rev. B 94, 155124 (2016).

[21] L. Huang, F. Wu, and J. Li, J. Chem. Phys. 144, 114708 (2016)

[22] W. Feng, X. Zhou, W. Q. Tian, W. Zheng, and P. A. Hu, Phys. Chem. Chem. Phys. 17, 3653 (2015).

[23] G. W. Mudd, S. A. Svatek, T. Ren, A. Patanè, O. Makarovsky, L. Eaves, P. H. Beton, Z. D. Kovalyuk, G. V. Lashkarev, Z. R. Kudrynskyi, and A. I. Dmitriev, Adv. Mater. 25, 5714 (2013).

[24] S. X. Yang, Y. Li, X. Z. Wang, N. J. Huo, J.-B. Xia, S.-S. Li, and J. B. Li, Nanoscale 6, 2582 (2014).

[25] A. Harvey, C. Backes, Z. Gholamvand, D. Hanlon, D. McAteer, H. C. Nerl, E. McGuire, A. Seral-Ascaso, Q. M. Ramasse, N. McEvoy, S. Winters, N. C. Berner, D. McCloskey, J. F. Donegan, G. S. Duesberg, V. Nicolosi, and J. N. Coleman, Chem. Mater. 27, 3483 (2015).

[26] P. Hu, L. Wang, M. Yoon, J. Zhang, W. Feng, X. Wang, Z. Wen, J. C. Idrobo, Y. Miyamoto, D. B. Geohegan, and K. Xiao, Nano Lett. 13, 1649 (2013).

[27] S. Lei, L. Ge, Z. Liu, S. Najmaei, G. Shi, G. You, J. Lou, R. Vajtai, and P. M. Ajayan, Nano Lett. 13, 2777 (2013).

[28] W. J. Jie, X. Chen, D. Li, L. Xie, Y. Y. Hui, S. P. Lau, X. D. Cui, and J. H. Hao, Angew. Chem., Int. Ed. 54, 1185 (2015).

[29] Z. X. Wang, K. Xu, Y. C. Li, X. Y. Zhan, M. Safdar, Q. Wang, F. M. Wang, and J. He, ACS Nano 8, 4859 (2014).

[30] X. Tao and Y. Gu, Nano Lett. 13, 3501 (2013).

[31] M. Lin, D. Wu, Y. Zhou, W. Huang, W. Jiang, W. S. Zheng, S. L. Zhao, C. H. Jin, Y. F. Guo, H. L. Peng, and Z. F. Liu, J. Am. Chem. Soc. 135, 13274 (2013).

[32] W. Huang, L. Gan, H. Li, Y. Ma, and T. Zhai, CrystEngComm 18, 3968 (2016).

[33] P. Hu, Z. Wen, L. Wang, P. Tan, and K. Xiao, ACS Nano 7, 5988 (2012).
[34] H. L. Zhuang and R. G. Hennig, Chem. Mater. 25, 3232 (2013).

[35] Y. Ma, Y. Dai, M. Guo, L. Yu, and B. Huang, Phys. Chem. Chem. Phys. 15, 7098 (2013).

[36] W. Wei, Y. Dai, C. Niu, X. Li, Y. Ma, and B. Huang, J. Mater. Chem. C 3, 11548 (2015).

[37] Y. Yoon, K. Ganapathi, and S. Salahuddin, Nano Lett. 11, 3768 (2011).

[38] P. E. Blöchl, Phys. Rev. B 50, 17953 (1994).

[39] J. P. Perdew, K. Burke, and M. Ernzerhof, Phys. Rev. Lett. 77, 3865 (1996).

[40] H. J. Monkhorst and J. D. Pack, Phys. Rev. B 13, 5188 (1976).

[41] G. Kresse and J. Hafner, Phys. Rev. B 47, 558 (1993).

[42] G. Kresse and J. Hafner, Phys. Rev. B 49, 14251 (1994).

[43] G. Kresse and J. Furthmüller, Comput. Mater. Sci. 6, 15 (1996).

[44] G. Kresse and J. Furthmüller, Phys. Rev. B 54, 11169 (1996).

[45] G. Henkelman, A. Arnaldsson, and H. Jonsson, Comput. Mater. Sci. 36, 354 (2006).

[46] J, Paier, M. Marsman, K. Hummer, G. Kresse, I. C. Gerber, and J. G. Angyan, J. Chem. Phys. 124, 154709 (2006).

[47] D. Alfe, Comput. Phys. Commun. 180, 2622 (2009).

[48] L. Huang, Z. Chen, and J. Li, RSC Adv. 5, 5788 (2015).

[49] V. Zolyomi, N. D. Drummond, and V. I. Fal'ko, Phys. Rev. B 87, 195403 (2013).

[50] V. Zolyomi, N. D. Drummond, and V. I. Fal'ko, Phys. Rev. B 89, 205416 (2014).

[51] H. Zheng, X. B. Li, N. K. Chen, S. Y. Xie, W. Q. Tian, Y. Chen, H. Xia, S. B. Zhang, and H. B. Sun, Phys. Rev. B 92, 115307 (2015).

[52] J. F. Nye, Physical Properties of Crystals (Clarendon, Oxford, 1985).

[53] C. Lee, X. Wei, J. W. Kysar, and J. Hone, Science 321, 385 (2008).

[54] M. Elahi, K. Khaliji, S. M. Tabatabaei, M. Pourfath, and R. Asgari, Phys. Rev. B 91, 115412 (2015).

[55] R. Saito, G. Dresselhaus, M. Dresselhaus, Physical Properties of Carbon Nanotubes (Imperial College Press, London, 2007).

[56] A. Politano, A. R. Marino, D. Campi, D. Farias, R. Miranda, and G. Chiarello, Carbon 50, 4903 (2012).

[57] L. J. Karssemeijer and A. Fasolino, Surf. Sci. 605, 1611 (2011).

[58] I. M. Lifshitz, Zh. Eksp. Teor. Fiz. 22, 475 (1952).

[59] P. Li and I. Appelbaum, Phys. Rev. B 92, 195129 (2015).

[60] T. Stauber, N. M. R. Peres, F. Guinea, and A. H. Castro Neto, Phys. Rev. B 75, 115425 (2007).

[61] D. Wickramaratne, F. Zahid, and R. K. Lake, J. Appl. Phys. 118, 075101 (2015).

[62] A. Pant, Z. Mutlu, D. Wickramaratne, H. Cai, R. K. Lake, C. Ozkan, and S. Tongay, Nanoscale 8, 3870 (2016).

[63] E. Aulich, J. L. Brebner, and E. Mooser, Phys. Status Solidi B 31, 129 (1969).

[64] J. Camassel, P. Merle, H. Mathieu, and A. Chevy, Phys. Rev. B 17, 4718 (1978). 